\title{
Maturation of the lower oesophageal sphincter in the preterm baby
}

\author{
S J NEWELL, P K SARKAR, G M DURBIN, I W BOOTH \\ AND A S MCNEISH
}

From the Institute of Child Health, University of Birmingham. Regional Neonatal Unit, Birmingham Maternity Hospital

SUMmARY There are few reported studies of the lower oesophageal sphincter in preterm infants and none has investigated babies of less than 34 weeks gestation. Using a modified manometric technique suitable for use on very low birth weight infants we have measured sphincter pressures on 68 occasions in 25 infants of postconceptional age between 27 and 41 weeks. In even the most preterm infants the lower oesophageal sphincter could be defined. The mean effective sphincter pressure rose from $3.8 \mathrm{mmHg}$ in infants of less than 29 weeks gestation to $18.1 \mathrm{mmHg}$ in the term infant. This rise in effective sphincter pressure correlated well with increasing postconceptional age $(r=0 \cdot 81)$. This pattern of maturation in our patients was unaffected by intrauterine growth retardation, postnatal illness, or concurrent xanthine administration.

In the preterm infant gastro-oesophageal reflux has been implicated in the pathogenesis of aspiration pneumonia, chronic lung disease, and apnoea.' It is also a significant risk factor for sudden infant death syndrome for which these infants are at high risk. ${ }^{2}$

The lower oesophageal sphincter plays an important role in the prevention of gastro-oesophageal reflux yet little is known about the maturation of this sphincter in the preterm infant. Previous studies used systems now considered unsatisfactory because of damped sensitivity to pressure change as a result of an inadequate pressure frequency response. This could explain the very low sphincter pressures that have been reported in children and infants. ${ }^{34}$ BoixOchoa studied infants of varying gestation, including preterm infants of 34 weeks and over. All infants were found to have no effective sphincter pressure until six weeks after birth. From this it was inferred that sphincter maturation is completely dependent on postnatal age and independent of gestation. ${ }^{4}$

Accurate systems are now available for use in older infants and children ${ }^{5}$ but the need for a small catheter and low perfusion flow rate precludes their use in preterm infants. Our aim was to devise a manometric system with a high sensitivity and low compliance

Address for correspondence: Dr S J Newell, Lecturer in Paediatrics, Institute of Child Health, The Nuffield Building, Francis Road, Birmingham B16 8ET.

Received for publication 31 July 1987. that would be suitable for use in infants of birth weights less than $1000 \mathrm{~g}$ and to study the resting lower oesophageal sphincter in the preterm very low birth weight infant in order to observe the early development of the sphincter and its subsequent pattern of maturation.

\section{Methods}

INFANTS

Twenty five infants were studied on 68 occasions. Mean birth weight was $1460 \mathrm{~g}$ (range: $690-3300 \mathrm{~g}$ ) and mean gestation $31 \cdot 2$ weeks (range 27-41 weeks); infants were studied between day one and nine weeks of age. Serial measurements were carried out on infants who remained on our neonatal unit until discharged home. This was not possible in those who were transferred back to their referring hospital when they no longer required intensive care.

Gestation at birth was assessed within two days of delivery by maternal history and Dubowitz assessment. ${ }^{6}$ Postconceptional age was calculated by adding the postnatal age to the gestation at birth.

The majority of measurements were made when infants were well and required only nursing care and tube feeding. None of those included in the study had clinical evidence of gastro-oesophageal reflux or other gastrointestinal disease. Six of the 25 infants studied 
were small for gestational age (19 measurements). Only nine of the measurements were made on infants who had not previously been fed and none of these was over one week of age, reflecting a policy of early enteral nutrition in our unit. Sixteen studies were carried out when infants were ill with clinical problems such as hyaline membrane disease, infection, and coagulopathy, and on seven occasions while these infants were receiving intermittent positive pressure ventilation. Nine of the studies on infants of less than 35 weeks postconceptional age were done while they were receiving a xanthine (caffeine or theophylline) for the treatment of apnoea. Xanthines were given in an individually tailored regime, adjusted to produce appropriate serum levels.

Informed consent was obtained from the parents; the protocol was approved by the Research Ethical Committee of the Central Birmingham Health Authority.

\section{MANOMETRIC SYSTEM}

We have developed a manometric system suitable for use in very low birth weight babies. Pressures were measured at the tip of a nasogastric tube attached to a fluid filled system with an inline pressure transducer. The system was continuously perfused at $3 \mathrm{ml} /$ hour with sterile $4 \%$ dextrose $/ 0 \cdot 18 \%$ saline delivered by a Vicker's IP3 high pressure pump through a stainless steel capillary tube (internal diameter $0.2 \mathrm{~mm}$ ) and then through the disposable pressure dome of an inline Bell and Howell pressure transducer (type 4327-I). The nasogastric tube, a standard feeding tube (Vygon 310.04, $1.5 \mathrm{~mm}$ external diameter, $0.9 \mathrm{~mm}$ internal diameter) modified to have a single end opening, was connected by a $100 \mathrm{~cm}$ extension tube (Vygon 1151-10). Dissolved gases were removed from the infusate under a partial vacuum before use.

The combination of a syringe pump capable of generating pressures over $1000 \mathrm{mmHg}$ and a capillary tube greatly reduces system compliance. This allows use of a low flow rate while still providing an adequate system response to pressure change. Occlusion of the catheter tip to flow resulted in a pressure rise of $50 \mathrm{mmHg} / \mathrm{s}$. This satisfies the requirements for accurate measurement of resting lower oesophageal sphincter pressure. ${ }^{7}$

The output of the transducer was amplified (Ormed isolated pressure amplifier type 4820 in preamplifier housing MT4PX) and fed to an Ormed 4 channel chart recorder (type MX412) running at 1 $\mathrm{mm} / \mathrm{s}$. Before and after each test, calibration was carried out with an electronic standard intrinsic to the amplifier and a mercury filled manometer. An error of $4 \%$ was accepted.

Non-disposable components were sterilised in a
$2 \%$ solution of activated glutaraldehyde (Cidex) for 24 hours and carefully rinsed with sterile water before and after use.

\section{INTRALUMINAL PRESSURE MEASUREMENTS}

The nasogastric tube was passed pernasally, and pressures measured at $0.5 \mathrm{~cm}$ intervals as the tip was advanced from the oesophagus through the lower oesophageal sphincter into the stomach. This was done twice and mean values taken. Measurements were made in this fashion so that at the end of each study, with minimal disturbance to the child, the catheter could be left in situ and used as a feeding tube. A pilot study was done on 16 infants in whom lower oesophageal sphincter pressure was measured using the antegrade technique and the retrograde slow pull through technique which is more commonly used in clinical practice. ${ }^{7}$ There was no significant difference between measurements made by either method. The mean difference between the two tests was $0.3 \mathrm{mmHg}$. The retrograde technique yielded slightly higher results, with $95 \%$ confidence limits of agreement between +2.3 and $-2.8 \mathrm{mmHg}{ }^{8}$

Studies were carried out at the time of a routine weekly change of nasogastric tube. The maximum interval between feeds in many of the infants was one hour. In order to approximate to the fasting state studies were done immediately before a feed.

The following pressures were measured: resting oesophageal pressure: the mean end expiratory pressure in the lower oesophagus before the sphincter. Fundal pressure: the mean end expiratory pressure in the fundus of the stomach immediately below the lower oesophageal sphincter. Actual sphincter pressure: the rise in pressure from the resting oesophageal pressure to the high pressure zone of the lower oesophageal sphincter. Effective sphincter pressure: the difference between the high pressure in the sphincter and the pressure measured in the fundus of the stomach; the pressure gradient responsible for the prevention of gastrooesophageal reflux.

STATISTICAL ANALYSIS

Correlation coefficients $r$ were calculated and probability inferred from the percentage points of the $t$ distribution. Tabulated data are presented as mean (SE).

\section{Results}

Oesophageal manometry was well tolerated by all infants, without apparent sequelae. Time taken for insertion of the nasogastric tube was between four and 22 minutes resulting in infusion of $0 \cdot 2-1 \cdot 1 \mathrm{ml}$ of solution during the procedure 
MANOMETRIC WAVEFORM

The principal features of the manometric pressure profile of the lower oesophageal sphincter in the preterm infant were the same as those described in adults, ${ }^{9}$ and children. ${ }^{4}$ Thus the position of the diaphragm could be identified by the change in the pattern of the superimposed respiratory waveform. As the catheter traverses the diaphragm the deflection during inspiration which is negative while the catheter is in the thorax becomes positive.

A rise in pressure, representing the lower oesophageal sphincter, was seen above and below the diaphragm. In the chest a small initial rise above the resting oesophageal pressure was followed, below the diaphragm, by a more marked rise in the high pressure zone of the sphincter. The initial rise in pressure above the diaphragm was small in the very preterm baby and more distinct as the baby became more mature.

The length of the high pressure zone of the lower oesophageal sphincter increased with postconceptional age from a mean of $6.5 \mathrm{~mm}(0.96)$ in the very preterm infant to $10 \mathrm{~mm}(1 \cdot 1)$ at term.

\section{INTRALUMINAL PRESSURES}

Consistent changes were seen in the lower oesophageal sphincter pressures with increasing maturity. Mean values, calculated from combined crosssectional and longitudinal data for effective sphincter pressure, actual sphincter pressure, resting oesophageal pressure and fundal pressure are shown in the Table. There was a marked rise in actual sphincter pressure and the effective sphincter pressure with a four fold increase between 27 and 40 weeks postconceptional age. Resting oesophageal pressure did not change and fundal pressure doubled.

There was a strong correlation between postconceptional age and both effective sphincter pressure $(r=0.81, p<0.001)$ (Fig. 1) and actual

Table Relationship between intraluminal pressures and postconceptional age in all infants studied

\begin{tabular}{|c|c|c|c|c|c|c|}
\hline & \multicolumn{6}{|c|}{ Postconceptional age (weeks) } \\
\hline & $27-28$ & $29-30$ & $31-32$ & $33-34$ & $35-36$ & $37+$ \\
\hline \multirow{3}{*}{$\begin{array}{l}\text { n } \\
\text { R O P }\end{array}$} & 4 & 13 & 15 & 16 & 12 & 8 \\
\hline & $2 \cdot 3$ & 3.0 & $3 \cdot 3$ & 3.0 & $2 \cdot 7$ & $3 \cdot 0$ \\
\hline & $(0 \cdot 9)$ & $(0 \cdot 7)$ & $(0 \cdot 6)$ & $(0 \cdot 5)$ & $(0 \cdot 5)$ & $(0 \cdot 6)$ \\
\hline \multirow[t]{2}{*}{ E S P } & 3.8 & $6 \cdot 1$ & $8 \cdot 5$ & $10 \cdot 3$ & $12 \cdot 2$ & $18 \cdot 1$ \\
\hline & $(0 \cdot 5)$ & $(0 \cdot 5)$ & $(0 \cdot 8)$ & $(0 \cdot 8)$ & $(1 \cdot 0)$ & $(1 \cdot 0)$ \\
\hline \multirow[t]{2}{*}{ A S P } & 5.0 & 8.9 & $12 \cdot 1$ & 13.7 & $16 \cdot 6$ & 22.9 \\
\hline & $(0 \cdot 7)$ & $(0 \cdot 7)$ & $(1 \cdot 2)$ & $(0.7)$ & $(1 \cdot 2)$ & $(1 \cdot 7)$ \\
\hline \multirow[t]{2}{*}{ F P } & 3.5 & 5.8 & 6.9 & 6.4 & $7 \cdot 1$ & $7 \cdot 8$ \\
\hline & $(1 \cdot 0)$ & $(0 \cdot 9)$ & $(0 \cdot 9)$ & $(0 \cdot 6)$ & $(0 \cdot 5)$ & $(0 \cdot 6)$ \\
\hline
\end{tabular}

Pressures $(\mathrm{mmHg})$ are given as mean (SE). ROP = resting oesophageal pressure; ESP = effective sphincter pressure; $\mathrm{ASP}=$ actual sphincter pressure $; \mathrm{FP}=$ fundal pressure .

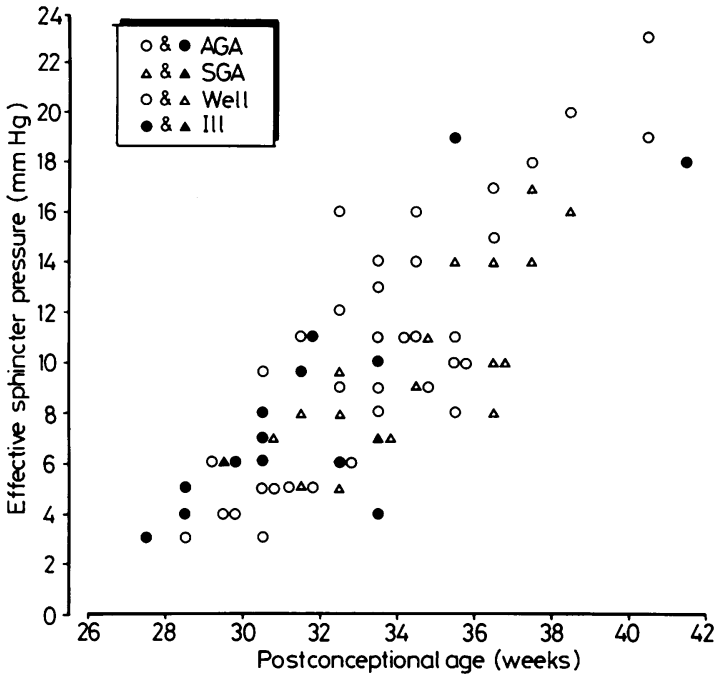

Fig. 1 Effective sphincter pressure and postconceptional age. $(r=0 \cdot 81, p<0.001 n=68)$ Circles $=$ appropriate for gestational age; Triangles = small for gestational age; open symbols = 'well'; closed symbols = 'ill'.

sphincter pressure $(\mathrm{r}=0 \cdot 80)$. Although effective sphincter pressure rose with postnatal age the correlation was less good $(r=0 \cdot 44)$ (Fig. 2).

Serial measurements were obtained from seven infants who were studied on four or more occasions. These infants were well and, although two had birth weights below the 10th centile, all were receiving full feeds and showing good weight gain. The longitudinal data (Fig. 3) confirm in individuals the trend seen in the combined results.

INFLUENCE OF INTRAUTERINE GROWTH, ILLNESS, AND XANTHINE ADMINISTRATION Effective sphincter pressure was unaffected by weight for gestational age, and perhaps surprisingly, by illness at the time of study (Fig. 2). Those infants receiving xanthines showed no tendency towards reduced effective sphincter pressure and had pressures which were not significantly different from other infants of similar postconceptional age $(p>0 \cdot 5)$.

\section{Discussion}

The successful development of a manometric technique for use in very low birth weight infants has allowed us to study the resting lower oesophageal sphincter in this group for the first time. There is a rise in effective sphincter pressure from 27 weeks postconceptional age to term. This rise is shown in the combined data and confirmed in individual infants who were followed longitudinally. 


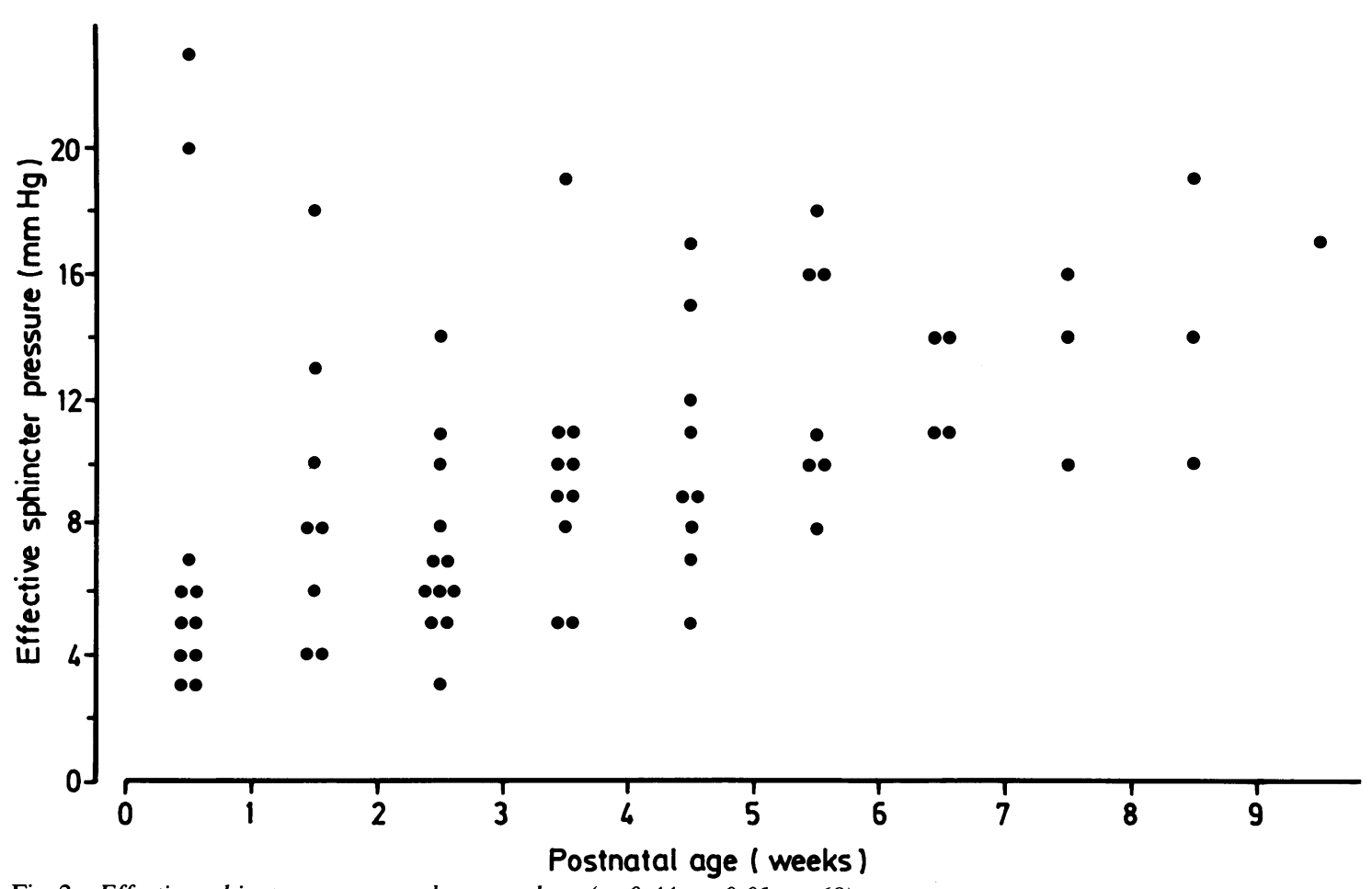

Fig. 2 Effective sphincter pressure and postnatal age $(r=0 \cdot 44, p<0 \cdot 01, n=68)$.

A low flow rate is mandatory when a perfused manometric system is used to measure lower oesophageal sphincter pressures in very low birth weight infants. Reduction of flow rate, however, can produce a slower pressure response, making quantitative pressure recording difficult. The

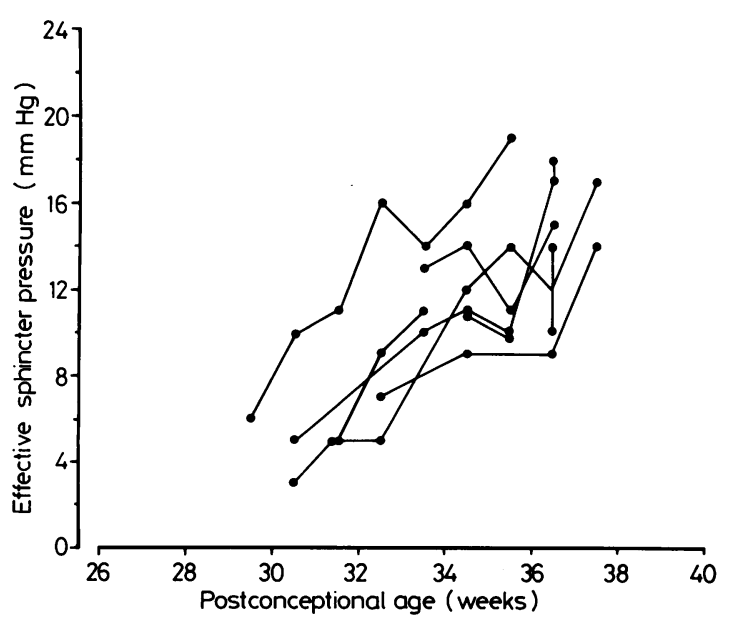

Fig. 3 Longitudinal studies. Effective sphincter pressure and postconceptional age. Each line represents sequential measurements made on one infant. $(n=7)$. combination of a high pressure pump and the high resistance capillary tubing provides a system with low compliance and an excellent dynamic pressure response. The removal of dissolved gases under a partial vacuum prevented the appearance of bubbles which might have greatly reduced pressure response. Inaccurate measurements will be made unless the defined requirements of a manometric system for intraluminal pressure measurements are met. ${ }^{7}$

A single lumen catheter with an end hole was used. This provides ease and reliability of manufacture and accurate measurements may be made. ${ }^{10}$ Radial asymmetry of lower oesophageal sphincter pressure will not be observed with such a system. Within the contraints of a single lumen tube, therefore, the use of an end hole design may be preferable because inaccuracies caused by pressure asymmetry are most likely to occur when a single side hole is used. ${ }^{?}$

The maturation of the lower oesophageal sphincter has several features which are seen in the crosssectional and longitudinal data. The main feature is a marked increase in the effective and actual sphincter pressures caused by a rise in pressure in the high pressure zone of the sphincter situated immediately below the diaphragm. This increase in sphincter pressure occurs as a function of the number of weeks 
since conception and is not affected by the time of birth. The strong correlation between effective sphincter pressure and postconceptional age (Fig. 1) is such that a baby born at 30 weeks gestation who is five weeks old will have the same effective sphincter pressure as a newborn baby of 35 weeks gestation.

These findings differ from the observations of Boix-Ochoa et al, ${ }^{4}$ who studied infants of 34 weeks postconceptional age or greater. In their study, sphincter pressures were lower; the rise in pressure correlated with postnatal age but was independent of gestation. No effective sphincter pressure was found in infants before six weeks of age. Once present, however, effective sphincter pressure did not exceed $1.5 \mathrm{mmHg}$. This finding in term infants at eight weeks of age differs considerably from more recently published data where mean effective sphincter pressures varied from $11-43 \mathrm{mmHg}{ }^{511-13}$ BoixOchoa's observation of much lower pressures may relate to methodological problems because, although a perfused system was used, no mention is made of perfusion rate or the system response to pressure change.

The contrast between our findings and those of previous workers is further emphasised on comparison of postnatal age and effective sphincter pressure (Fig. 2). Here not only is the correlation coefficient poor $(0.44)$, but also some of the highest pressures we found were in full term infants who were studied within a few days of birth.

We also observed that from the first days after birth even the very preterm infants of less than 30 weeks gestation were able to produce a lower oesophageal sphincter pressure higher than the fundal pressure, providing a definite, albeit small, pressure gradient between stomach and oesophagus. In these and all other infants the fundal pressure was higher than the resting oesophageal pressure, and thus this small pressure gradient may be important in preventing continuous gastro-oesophageal reflux.

We noted that sphincter length increased with maturity. The trend was clear although exact values should be interpreted with caution because our open ended catheter was not specifically designed for the measurement of sphincter length.

Infants with prenatal intrauterine growth retardation or postnatal illness had results within the distribution of the combined cross-sectional and longitudinal data (Fig. 1). The infants on whom serial measurements were made were well, although two were born small for gestational age, and, therefore, further longitudinal studies are required to determine the effect of more longterm illness or growth retardation. Furthermore because the full spectrum of neonatal disease was not represented the possibility of altered sphincter maturation as a result of illness cannot be excluded. The lack of apparent effect of these factors upon sphincter maturation, however, was surprising and differs from some other aspects of early gut development. ${ }^{14} 15$

Xanthines have previously been shown to reduce the lower oesophageal sphincter pressure ${ }^{16}$ and increase the amount of gastro-oesophageal reflux in term infants at risk of sudden infant death syndrome. ${ }^{17}$ In this study infants receiving xanthines had results which did not differ from the general distribution. Numbers were small, however, and further longitudinal studies are required to more clearly define the effect of xanthines on the preterm sphincter.

Finally those infants who had not been fed had sphincter pressures within the general distribution. All these infants were less than seven days old because of our policy of early enteral nutrition of very preterm infants with expressed breast milk.

We have shown that a potential antireflux mechanism exists in infants from 27 weeks gestation and that the mechanism probably becomes more effective as the infant matures. The exact relationship between effective sphincter pressure and gastro-oesophageal reflux, and between these two factors and putative reflux related disorders such as recurrent apnoea, however, remains largely unexplored.

Simon Newell was sponsored by the National Fund for Research into Crippling Diseases (Action Research) and gratefully acknowledges their generous support. We are indebted to $\mathrm{Mr} \mathrm{G} \mathrm{W}$ Hollins and $\mathrm{Mr} \mathrm{P} \mathrm{H}$ Williams of the Medical Physics Department of the Birmingham Maternity Hospital for their technical expertise and to the medical and nursing staff of the Neonatal Unit for their cooperation. (This paper has previously been published in abstract form following the XIX Annual Meeting of the European Society for Paediatric Gastroenterology and Nutrition (ESPGAN): Pediatr Res 1986; 20: 692.)

\section{References}

1 Herbst JJ, Minton SD, Book LS. Gastroesophageal reflux causing respiratory distress and apnoea in newborn infants. $J$ Pediatr 1979; 95: 763-8.

2 Leape LL, Holder TM, Franklin JD. Respiratory event in infants secondary to gastroesophageal reflux. Pediatrics 1977; 60: 924-7.

3 Gryboski JD. The swallowing mechanism of the neonate. Esophageal and gastric motility. Pediatrics 1965; 35: 445-51.

4 Boix-Ochoa J, Canals J. Maturation of the lower oesophagus. J Pediatr Surg 1976; 11: 749-56.

5 Euler AR, Ament ME. Value of esophageal manometric studies in the gastroesophageal reflux of infancy. J Pediatr 1977; 59: 58-61. 
6 Dubowitz LMS, Dubowitz V, Goldberg C. Clinical assessment of gestational age in the newborn infant. J Pediatr 1970; 77: 1-10.

7 Weihrauch TR. Oesophageal manometry: methods and clinical practice. Baltimore-Munich: Urban and Schwarzenberg, 1981: 20-63.

8 Bland JM, Altman DG. Statistical methods for assessing agreement between two methods of clinical measurement. Lancet 1986; i: 307-10.

9 Winans CS, Harris LD. Quantitation of lower esophageal sphincter competence. Gastroenterology 1967; 52: 773-8.

10 Pope CE. A dynamic test of sphincter strength: its application to the lower esphageal sphincter Gastroenterology 1967; 52: 779-86.

11 Kenigsberg K, Aiges H, Alperstein G. A unique device to measure lower esophageal sphincter pressure in unsedated infants. J Pediatr Surg 1981; 16: 370-73.
12 Euler AR, Byrne WJ. Twenty four hour esophageal intraluminal $\mathrm{pH}$ probe testing: a comparative analysis. Gastroenterology 1981; 80: 957-61.

13 Moroz SP, Espinoza J, Cumming WA, Diament NE. Lower esophageal sphincter function in children with and without gastroesophageal reflux. Gastroenterology 1976; 71: 236-41.

14 Mayne AJ, Brown GA, Sule D, McNeish AS. The postnatal development of disaccharidase activities in jejunal fluid of pre-term neonates. Gut 1986; 27: 1357-61.

15 Bisset WM, Watt J, Rivers R, Milla P. The ontogeny of small intestinal motor activity. Pediatr Res 1986; 20: 692.

16 Dennish GW, Castell DO. Caffeine and the lower esophageal sphincter. Am J Dig Dis 1972; 17: 993-996.

17 Vandenplas Y, De Wolf D, Sacre L. Influence of xanthines on gastroesophageal reflux in infants at risk for sudden infant death syndrome. Pediatrics 1986; 77: $807-10$. 